\title{
INVESTIGATION OF COPPER CORROSION INHIBITION WITH FREQUENCY-DEPENDENT ALTERNATING-CURRENT SCANNING ELECTROCHEMICAL MICROSCOPY
}

\author{
J.J. Santana ${ }^{1,+}$, M. Pähler², W. Schuhmann², R.M. Souto ${ }^{1,3}$ \\ ${ }^{1}$ Department of Physical Chemistry, University of La Laguna, E-38200 La Laguna, \\ Tenerife, Canary Islands, Spain \\ ${ }^{2}$ Analytische Chemie - Elektroanalytik \& Sensorik; Ruhr-Universität Bochum, \\ Universitätsstraße 150, D-44780 Bochum, Germany \\ ${ }^{3}$ Instituto de Materiales y Nanotecnologías, University of La Laguna, E-38200 La \\ Laguna, Tenerife, Canary Islands, Spain \\ + On leave from the Department of Process Engineering, University of Las Palmas de \\ Gran Canaria, Campus Universitario de Tafira, E-35017 Las Palmas de Gran Canaria, \\ Canary Islands, Spain.
}

\begin{abstract}
:
Alternating current scanning electrochemical microscopy (AC-SECM) is used to investigate the inhibition of copper corrosion by four model organic molecules, namely benzotriazole (BTAH), 5-methyl-benzotriazole (MBTAH), 2-mercaptobenzimidazole (MBI), and ethyl xanthate (EX). It is shown that the formation of inhibitor films on the metal can be followed from the decrease of the substrate's conductivity associated with the formation of inhibitor-containing surface layers. Very sensitive 3D imaging can also be performed that accounts for changes in chemical reactivity of the modified surfaces, as well as for their corrosive attack from the environment. AC-SECM is considered a powerful technique for the investigation of corrosion processes.
\end{abstract}

Keywords: alternating current scanning electrochemical microscopy; corrosion inhibition; copper; benzotriazole; benzimidazole; ethyl xanthate. 


\section{Introduction}

Scanning electrochemical microscopy has quickly become an important tool for the in situ investigation of corrosion processes in electrolyte solutions detecting localized electrochemical activities with spatial resolution [1-3]. Spatially resolved imaging has been achieved in solution for the detection of precursor sites for pitting corrosion [4-7], the imaging of metastable pits for the first time [8], the monitoring of single pits nucleation and growth [9], the characterization of galvanic coupling [10], the formation of inhibitor films on active metals [11-13], and the detection of electrochemical reactions occurring at defects in coated metals where the underlying metal was directly exposed to the electrolyte $[14,15]$. The characterization of surface chemical reactivity by SECM is not restricted to the case of metal electrodes, because it has also been successfully employed with materials coated by highly unreactive inorganic layers such as alumina [16,17]. Details of heterogeneous electrolyte uptake by metal-coated metals due to a specific effect of chloride ions were revealed through the observation of SECM images [18-20], and the growth of single blisters could even be monitored at very early exposures [21].

Imaging in scanning electrochemical microscopy is performed by measuring a faradaic current at the ultramicroelectrode probe (UME). When a diffusion-controlled reversible redox reaction is used as electrochemical mediator, the current measured at the tip is affected by the nature of the surface under investigation and the tip-substrate distance. Separate control of the electric state of the substrate can be attained by using a bipotentiostat as electrochemical interface in the system, allowing for the controlled polarization of the sample. Nevertheless, operation of the system under open circuit conditions is of a great advantage, especially when naturally corroding conditions are explored. Indeed, such state can be easily guaranteed when the SECM is operated in generation-collection modes, whereas it is not directly evident when operation in the feedback mode is performed instead. In this case, an external electrochemical mediator is added to the system for chemical imaging purposes, which should be a reversible redox couple. But the potential of the system will be determined by the ratio between the two species involved in the redox couple, and can be established by applying the Nernst equation. Thus, polarization of the system is effectively produced by the electrochemical mediator. Additionally, the possible interference of any of the redox species on the actual corrosion process under investigation cannot be discarded, 
particularly when thin surface films, such as those formed during the adsorption of corrosion inhibitors on metals, are involved.

This limitation of scanning electrochemical microscopy can be overcome by using a different technique, frequency-dependent alternating current scanning electrochemical microscopy (AC-SECM) [22], which does not require the addition of electrochemical mediators, though employing the same ultramicroelectrodes than in conventional SECM. The concept of a variation in impedance upon approaching an UME to the sample surface had first been used for precise vertical positioning of SECM tips when amperometric [23], enzymatic [24] or potentiometric [25] sensors were employed as tips. The alternating current response may be successfully used to monitor variations in the local electrochemical activity of a sample [26-30], which represents a powerful tool for the analysis of inhibitor-metal systems where a homogeneous sample surface is provided [31]. In these systems, a negative feedback, positive feedback, or both responses can be observed [29,31]. Up to now, this technique has been successfully employed in the corrosion laboratory to detect corrosion pits [32-34], to monitor metal release [35,36], and to visualize surface inhomogeneities in polymercoated metals related to the specific penetration of chloride ions through the organic film [37].

In this paper we report on the applicability of frequency-dependent AC-SECM to investigate the inhibition of metal corrosion. To demonstrate the feasibility of this approach, $\mathrm{Cu}$ surfaces partially modified with different organic compounds, namely benzotriazole (BTAH), 5-methyl-benzotriazole (MBTAH), 2-mercaptobenzimidazole (MBI), and ethyl xanthate (EX), were investigated by means of multi-frequency ACSECM. In general, corrosion inhibitors are compounds which form a thin passivating layer covering the metal surface they should protect by adsorption or chemisorption. BTAH is an established and widely explored inhibitor against copper corrosion [38]. It binds via chemisorption to $\mathrm{Cu}(\mathrm{I})$-centres located on the surface [39], and the formed layer has a thickness of only a few nanometres depending on the time the metal was immersed in the inhibitor solution. MBI has been found to be more effective than BTAH for the corrosion protection of copper in aerated $\mathrm{HCl}$ aqueous environment [40], and its inhibitive action is exerted mainly on the anodic process. In general terms, both MBI and BTAH are regarded to exhibit excellent inhibitor characteristics for corrosion protection in many aggressive environments [41]. Finally, the mineral collector EX has been observed to offer high protection against copper corrosion in neutral and 
moderately alkaline aqueous $\mathrm{NaCl}$ solutions [42,43]. XPS data suggest that copper protection is achieved through the growth of a cuprous diethyl dixanthogen layer on the metal in which the oxidation state +1 of copper becomes stabilized [44].

\section{Experimental}

Benzotriazole (BTAH; Avocado Research Chemicals Ltd., UK), 5-methyl-1Hbenzotriazole (MBTAH; Aldrich, USA), 2-mercaptobenzimidazole (MBI; Aldrich, UK), and potassium ethyl xanthate (EX; Fluka Chemica, Switzerland) were used as received. Potassium chloride (KCl; Riedel de Haën, Germany) and sodium sulphate ( $\mathrm{Na}_{2} \mathrm{SO}_{4}$; J.T. Baker, The Netherlands) were analytical grade quality. Inhibitor solutions were prepared at a $1 \mathrm{mM}$ concentration by dissolving the corresponding inhibitor in a $100 \mathrm{mM} \mathrm{KCl}$ solution. All aqueous solutions were prepared using ultra-pure water purified with a Milli-Q system from Millipore.

Measurements were performed on 99.99\% copper purity plates (Goodfellow, UK) cut to dimensions $3 \mathrm{~cm} \times 3 \mathrm{~cm}$ and $1 \mathrm{~mm}$ of thickness. The surface of the samples was ground to a 1500 grit finish, followed by two consecutive polishing steps with 1 and $0.3 \mu \mathrm{m}$ alumina slurries, respectively. The resulting surfaces were degreased with acetone, abundantly rinsed with ultra-pure deionised water and allowed to dry in air. Inhibitor films were produced on the copper electrode ex-situ by dipping the metallic samples in the inhibitor-containing solution for two different immersion times (5, and 45 min, respectively). On the other hand, the persistence of the inhibitor films deposited on copper was tested by exposing half of the sample to $3 \mathrm{wt} \% \mathrm{NaCl}$ solution for $5 \mathrm{~h}$. A sketch that shows the six different regions produced on a freshly polished copper sample following this procedure is shown in Figure 1. The dotted region in the figure denotes the portion of the sample that was immersed in the $\mathrm{NaCl}$ solution.

The set-up for AC-SECM and the procedures for SECM imaging in the AC mode have been previously described [34]. 25- $\mu$ m-diameter SECM tips were glassinsulated Pt micro-disk electrodes [47]. A three-electrode configuration was accomplished with a Pt-wire as counter electrode and a $\mathrm{Ag} / \mathrm{AgCl}$ pseudo reference electrode. The frequency range applied ranged from 351 to $55493 \mathrm{~Hz}$ with a total number of points of 26 (logarithmic spaced). The AC signal had a potential amplitude of $0.1 \mathrm{~V}$ and a sensibility of $200 \mathrm{mV} . X$ and $Y$ tilt corrections during imaging of planar substrates have been implemented such that the $(Z)$ piezo movement accounts for the defined substrate slopes at each point of an image. The entire system is placed on an 
vibration isolation table and is enclosed in a Faraday cage. For a predetermined perturbing voltage and tip-to-sample distance, variations in the current magnitude $(R)$ and phase shift $(\theta)$ throughout line scans reflect changes in the local electrochemical activity of the sample, with greater electrochemical activity areas typically displaying bigger values of $R$ and $\theta$, respectively. AC-SECM measurements were performed in 1 $\mathrm{mM} \mathrm{Na}_{2} \mathrm{SO}_{4}$ solution, open to air, and maintained at room temperature.

\section{Results and discussion}

In the theory of amperometric SECM, it is established that different trends are found in the approach curves depending on whether an insulating (negative feedback) or a conductive (positive feedback) surface is monitored [48]. Topographic images of surfaces could thus be obtained. In the case of AC-SECM, in addition to the topographic characterization derived from the experimental variation of the AC response with the tip-substrate distance, spatially-resolved information on the chemical and electrochemical reactivity of a surface can be obtained too [22]. The major advantage is that no redox mediator is required in this case, thus avoiding eventual chemical interferences with the substrate to take place. Chemical and electrochemical sensitivity are due to the changes in the AC current distribution in the electrochemical cell that occur when the microelectrode is placed in the proximity of a surface [28,34]. Depending on the electrochemical characteristics of the portion of the investigated surface directly under the tip, the AC current will preferentially flow through one of the pathways depicted in Figure 2 according to their corresponding impedances [34].

A negative feedback behaviour is always observed in the approach curves, regardless the frequency of the AC excitation signal, for an insulating surface. Conversely, both negative and positive feedback curves are determined in the case of active surfaces. The negative feedback behaviour is found in the low frequency range, and the positive feedback is found at higher frequency values. A transition between negative and positive feedback occurs at an intermediate frequency value, and this value is shifted in the high frequency direction for less conductive substrates. On the other hand, an optimum AC frequency value may be found for which the greatest electrochemical contrast can be achieved for chemical imaging [31]. The actual experimental procedure involves a series of steps as described next. First, approach curves are measured in a wide frequency range at various locations of the surface. That is, a frequency spectrum is applied at each point of the approach curve whereas the tip is 
moved in the $Z$ direction towards the substrate. When the tip reaches the position of closest approach, the approach curves at each excitation frequency can be composed. For the sake of illustration, Figure 3 show a selection of the approach curves determined above three different regions formed on a copper sample that was modified with BTAH; they correspond to bare copper, $\mathrm{Cu} / \mathrm{BTAH}$ treated for $5 \mathrm{~min}$, and $\mathrm{Cu} / \mathrm{BTAH}$ treated for 45 min. The BTAH-modified regions were produced by dipping a freshly polished copper sample in the inhibitor-containing solution for less than half of its length from either side for the chosen pre-treatment durations, leaving the untreated copper zone in the centre. In general, the formation of the inhibitor films on the copper sample originates less conductive surfaces, which are characterized by a frequency range of the approach curves with negative feedback behaviour that extends over most of entire frequency range covered in this work (cf. Figure 3 for the case of the copper sample treated with BTAH). In this way, the transition frequency between negative and positive feedback is shifted to higher frequency values as result of the formation of thicker inhibitor films on the metal for the longer pre-treatments, a result that agree well with our previous reports [31]. This is a direct observation of changes in the time constant corresponding to the different pathways given in the simplified equivalent circuit of Figure 2, which is the result of the more insulating characteristics of the surface due to its coverage by the inhibitor.

It is interesting to notice that the magnitude of the positive feedback effect is significantly smaller for the inhibitor pre-treated samples compared to that untreated even at the highest frequency under consideration, though positive feedback behaviour was observed for all the systems (cf. Figures 3 and 4). The normalized magnitude of the current was always smaller than 1.4 at the position of closest approach for all the tested systems, with the only exception of the sample treated with EX for $5 \mathrm{~min}$, when a value close to 1.8 was measured. The later is an indication of slower adsorption kinetics of this molecule on copper, though this suggestion needs further work to be confirmed. Certainly these values are significantly smaller than the normalized magnitude values close to 2.1 measured for the untreated copper sample, which correspond with a conductive substrate. In particular, the coverage is very high already for the shortest pre-treatment with BTAH as shown by the smaller magnitude currents than on the untreated copper metal. The existence of a zone in the sample with untreated metal allows to attribute totally the decrease of conductivity to the formation of inhibitor films on the metal surface and not to metal oxide development. These results are also in 
agreement with those previously observed on polarized copper samples by conventional amperometric SECM, where the polarization of the metal did not allow oxide to be formed on it [17].

Next, the AC-SECM image of the substrate to be investigated could be obtained by moving the tip in a plane $X-Y$ defined over the surface to achieve a constant tipsubstrate distance. Dynamic tip-height correction was performed in the system to compensate for any unavoidable tilt present in the sample. The instrument can perform such correction automatically on the basis of the previous measurement of three approach curves at different points of the surface. The software stores the value of the $Z$-direction for each of the three positions, then calculates the coordinates of the plane that contains these positions, and it can dynamically follow the tilt of the sample to maintain constant tip-substrate( plane) distance throughout the experiment. The approaches were performed on the various inhibitor-treated surfaces and not above the untreated copper zones. The working distance was established at $12 \mu \mathrm{m}$, which is approximately the tip radius.

Figures 6A-9A show the 3D images obtained using AC-SECM for copper with the four inhibitor systems considered in this work. They have been measured over an area of approximately $2000 \mu \mathrm{m} \times 1200 \mu \mathrm{m}$ around the centre of the samples, thus allowing the six regions of different chemical reactivity produced on their surface to be viewed in the same image. The untreated copper regions are located in the centre, whereas the portions of the surface that were treated in the inhibitor for the chosen pretreatment times are found at either side from the central strip. Additionally, the lower half of the 3D images show the portion of the surface exposed to an aggressive solution to produce modification of the surface films on the metals and the corrosive attack eventually. In this way, these six regions can be compared to account for both the extent of the inhibiting action provided by the films obtained for different pre-treatment durations, and the persistence of those films against the corrosive attack to the metal substrate that occurs in $3 \mathrm{wt} \% \mathrm{NaCl}$ solution. In the next paragraphs, each of the copperinhibitor systems will be considered in turn on the basis of the information supplied by the AC-SECM technique.

The different chemical reactivity of the six regions produced in a copper specimen treated with BTAH are viewed as zones of different values in the magnitude of the AC current signal recorded at the SECM tip when travelling above the sample at a constant height as depicted in Figure 6A. A strip displaying the higher current 
magnitude values is found at the centre of the image extending from top to bottom, which matches the portion of the copper surface that was not treated with BTAH. But even in this strip, differences can be observed between the region of the bare copper that was subsequently exposed to the chloride-containing solution for 5 hours and the other portion that was not immersed in the electrolyte. And the highest values are measured over the later. Smaller current magnitudes correspond to less conductive substrates, thus indicating that some passivation of the copper surface occurs during the exposure to the chloride solution. On the other hand, the areas that have been treated with BTAH show smaller current magnitudes in the 3D image, though differences in their magnitudes are observed between the regions pre-treated with the inhibitor molecule for different durations, and also with those that were immersed in 3wt.\% $\mathrm{NaCl}$. These features are better observed in Figure 6B which has been built extracting two line scans along the $X$ axis (i.e. for the tip travelling from left to right in the 3D image) from the image in Figure 6A. In this way, current magnitude values both from the portion of the sample that was exposed to the chloride-containing solution and from that non-exposed could be plotted. BTAH is observed to protect the surface of copper by forming surface films which are less conductive than the bare metal, this effect being significantly bigger in the case of the longer pre-treatment of 45 minutes. This compact and more stable film is not affected during exposure to the chloride solution, as revealed by the same current magnitudes found in both regions. Conversely, the surface film formed on copper by dipping the metal in the BTAH solution for 5 minutes exhibits rather poor protective characteristics, and only a small reduction in the measured values is observed this time. Yet, the performance of this surface film is notably improved during the exposure to the chloride-containing solution, leading to the formation of a surface film of similar characteristics as that originally formed during the longer pre-treatment with BTAH. In summary, a more protective inhibitor film has been obtained for this organic molecule during exposure to the chloride solution, either because chloride ions were included in the inhibitor film, or due to additional thickening of the passive oxidecontaining film on copper during formation of more protective inhibitor films in the case of this organic molecule.

The faster adsorption of MBTAH on copper is easily observed from the corresponding AC-SECM plots given in Figure 7. Within experimental error, the same current magnitude values are measured over the regions treated with this inhibitor for either 5 or 45 minutes, and the outstanding sealing characteristics of these films are only 
slightly enhanced during the exposure to the chloride-containing environment. Furthermore, the difference in the values measured over the inhibitor-protected and the bare metal surface is bigger than in the case of BTAH, which correlates well with the bigger inhibition efficiency conferred to copper by MBTAH.

A rather fast adsorption of the inhibitor on copper also occurs in the case of MBI. Now, only slightly bigger current magnitude values are measured in the case of the shorter pre-treatment (see Figure 8). In addition, significant improvement of the protective characteristics of the surface films also occurs for this inhibitor when they were exposed to the chloride-containing solution.

The distinctive behaviour of ethyl xanthate for the corrosion protection of copper compared to the other three inhibitors is also observed in the AC-SECM results presented in Figure 10. Long exposures to an EX-containing solution are needed to form on copper the best protective film, and this film is only slightly affected by chloride ions. Conversely, the film formed by dipping the metal in the inhibitor solution for 5 minutes is very weak, and it is removed from the surface of the metal during exposure to the chloride-containing solution. In this case, no clear difference exists between the regions of untreated copper and that previously treated with the inhibitor.

From the foregoing, a sequence of inhibition efficiencies provided by the different inhibitors can be established which agrees well with the results in the literature [55,56], and the controversy existing in the case of ethyl xanthate as corrosion inhibitor for copper has been resolved. This compound requires long pre-treatments in order to form compact protective films on copper, but these provide the greatest protection once formed [51]. On the other hand, shorter pre-treatments, similar in duration to those required for the other inhibitors considered in this work to form protecting surface films on this metal, do not lead to the formation of a compact EX-containing film and thus no effective protection is provided to copper.

\section{Conclusions}

Frequency-dependent alternating-current scanning electrochemical microscopy allows us to distinguish and quantify the protection characteristics of inhibitor films formed on metals, as well as the stability of those films in aggressive environments.

The current magnitude values measured at the SECM tip can be plotted to produce 3D images which allow differences in chemical reactivity in the modified surfaces to be detected. Enhanced chemical contrast can be obtained by adequately 
choosing the frequency of the AC perturbation signal employed for imaging. Approach curves towards the sample can be employed to assist that choice.

The versatility of this technique to investigate samples containing zones with different pre-treatments and aggressive attacks for inhibitor screening is due to the use of a highly diluted electrolyte without any redox mediator which might influence the local chemistries at the metal surface.

\section{Acknowledgements:}

The authors are grateful to the German Academic Exchange Service (DAAD, Bonn) and to the Spanish Ministry of Science and Innovation (MICINN, Madrid, Acción Integrada No. HA2006-0077) for the grant of a Collaborative Research Programme between Germany and Spain. M.P. and W.S. are grateful for financial support by the DFG in the framework of the SFB459 (A5) and the project "Elucidation of corrosion phenomena with high lateral resolution using scanning probe techniques" (SCHU 929/9-1). J.J.S. and R.M.S. are grateful for financial support to the Ministry of Science and Innovation (Madrid, Spain) and the European Regional Development Fund (Brussels, Belgium) in the framework of Project CTQ2009-12459.

\section{References:}

1. S.E. Pust, W. Maier, G. Wittstock, Investigation of localized catalytic and electrocatalytic processes and corrosion reactions with scanning electrochemical microscopy (SECM), Z. Phys. Chem. 222 (2008) 1463-1517.

2. L. Niu, Y. Yin, W. Guo, M. Lu, R. Qin, S. Chen, Application of scanning electrochemical microscope in the study of corrosion of metals, J. Mater. Sci. 44 (2009) 4511-4521.

3. R.M. Souto, S. Lamaka, S. González. Uses of scanning electrochemical microscopy in corrosion research. In: "Microscopy: Science, Technology, Applications and Education”, Vol. 3 (A. Méndez-Vilas, J. Díaz, eds.). Formatex Research Center, Badajoz (Spain), 2010, pp. 1769-1780.

4. Y.Y. Zhu, D.E. Williams, Scanning electrochemical microscopic observation of a precursor state to pitting corrosion of stainless steel, J. Electrochem. Soc. 144 (1997) L43-L45. 
5. L.F. Garfias-Mesias, M. Alodan, P.I. James, W.H. Smyrl, Determination of precursor sites for pitting corrosion of polycrystalline titanium by using different techniques, J. Electrochem. Soc. 145 (1998) 2005-2010.

6. S.B. Basame, H.S. White, Scanning electrochemical microscopy of metal/metal oxide electrodes. Analysis of spatially localized electron-transfer reactions during oxide growth, Anal. Chem. 71 (1999) 3166-3170.

7. I. Serebrennikova, S. Lee, H.S. White, Visualization and characterization of electroactive defects in the native oxide film on aluminium, Faraday Discuss. 121 (2002) 199-210.

8. Y. González-García, G.T. Burstein, S. González, R.M. Souto, Imaging metastable pits on austenitic stainless steel in situ at the open-circuit corrosion potential, Electrochem. Commun. 6 (2004) 637-642.

9. C. Gabrielli, S. Joiret, M. Keddam, N. Portail, P. Rousseau, V. Vivier, Single pit on iron generated by SECM. An electrochemical impedance spectroscopy investigation, Electrochim. Acta 53 (2008) 7539-7548.

10. A.M. Simões, A.C. Bastos, M.G. Ferreira, Y. González-García, S. González, R.M. Souto, Use of SVET and SECM to study the galvanic corrosion of an iron - zinc cell, Corros. Sci. 49 (2007) 726-739.

11. K. Mansikkamaki, P. Ahonen, G. Fabricius, L. Murtomaki, K. Kontturi, Inhibitive effect of benzotriazole on copper surfaces studied by SECM, J. Electrochem. Soc. 152 (2005) B12-B16.

12. K. Mansikkamaki, C. Johans, K. Kontturi, The effect of oxygen on the inhibition of copper corrosion with benzotriazole, J. Electrochem. Soc. 153 (2006) B22-B24.

13. J. Izquierdo, J.J. Santana, S. González, R.M. Souto, Uses of scanning electrochemical microscopy for the characterization of thin inhibitor films on reactive metals: the protection of copper surfaces by benzotriazole, Electrochim. Acta 55 (2010) 8791-8800.

14. R.M. Souto, Y. González-García, S. González, In situ monitoring of electroactive species by using the scanning electrochemical microscope. Application to the investigation of degradation processes at defective coated metals, Corros. Sci. 47 (2005) 3312-3323.

15. A. Simões, D. Battocchi, D. Tallman, G. Bierwagen, Assessment of the corrosion protection of aluminium substrates by a Mg-rich primer: EIS, SVET and SECM study, Prog. Org. Coat. 63 (2008) 260-266. 
16. D. Battistel, S. Daniele, G. Battaglin, M.A. Baldo, A simple electrochemical strategy for the characterisation of defects in alumina-coated metal substrates, Electrochem. Commun. 11 (2009) 2195-2198.

17. D. Battistel, S. Daniele, R. Gerbasi, M.A. Baldo, Characterization of metalsupported $\mathrm{Al}_{2} \mathrm{O}_{3}$ thin films by scanning electrochemical microscopy, Thin Solid Films 518 (2010) 3625-3631.

18. R.M. Souto, Y. González-García, S. González, G.T. Burstein, Damage to paint coatings caused by electrolyte immersion as observed in situ by scanning electrochemical microscopy, Corros. Sci. 46 (2004) 2621-2628.

19. R.M. Souto, Y. González-García, S. González, Characterization of coating systems by scanning electrochemical microscopy: surface topology and blistering, Prog. Org. Coat. 65 (2009) 435-439.

20. E. Salamifar, M.A. Mehrgardi, M.F. Mousavi, Ion transport and degradation studies of a polyaniline-modified electrode using SECM, Electrochim. Acta 54 (2009) 4638-4646.

21. R.M. Souto, Y. González-García, S. González, G.T. Burstein, Imaging the origins of coating degradation and blistering caused by electrolyte immersion assisted by SECM, Electroanalysis 21 (2009) 2569-2574.

22. K. Eckhard, W. Schuhmann, Alternating current techniques in scanning electrochemical microscopy (AC-SECM), Analyst 133 (2008) 1486-1497.

23. D.M. Osbourn, R. H. Sanger, P. J. S. Smith, Determination of single-cell consumption with impedance feedback for control of sample-probe separation, Anal. Chem. 77 (2005) 6999-7004.

24. B.R. Horrocks, D. Schmidtke, A. Heller, A. J. Bard, Scanning electrochemical microscopy. 24. Enzyme ultramicroelectrodes for the measurement of hydrogen peroxide at surfaces. Anal. Chem. 65 (1993) 3605-3614.

25. R. Kashyap, K. Gratzl, Adjusting the distance of electrochemical microsensors from secreting cell monolayers on the micrometer scale using impedance, Anal. Chem. 71 (1999) 2814-2820.

26. A. Schulte, S. Belger, W. Schuhmann, Localised electrochemical impedance spectroscopy with high lateral resolution by means of alternating current scanning electrochemical microscopy, Electrochem. Commun. 4 (2002) 134-138. 
27. M. Etienne, A. Schulte, W. Schuhmann, High resolution constant-distance mode alternating current scanning electrochemical microscopy (AC-SECM), Electrochem. Commun. 6 (2004) 288-293.

28. A.S. Baranski, P.M. Diakowski, Application of AC impedance techniques to scanning electrochemical microscopy, J. Solid State Electrochem. 8 (2004) 683-692.

29. P.M. Diakowski, A.S. Baranski, Positive and negative AC impedance feedback observed above conductive substrates under SECM conditions, Electrochim. Acta 52 (2006) 854-862.

30. P.M. Diakowski, S. Ding, Novel strategy for constant-distance imaging using alternating current scanning electrochemical microscopy, Electrochem. Commun. 9 (2007) 2617-2621.

31. M. Pähler, J.J. Santana, W. Schuhmann, R.M. Souto, Application of AC-SECM in corrosion science - Local visualisation of inhibitor films on active metals for corrosion protection, Chem. Eur. J. 17 (2011) 905-911.

32. B.B. Katemann, C.G. Inchauspe, P.A. Castro, A. Schulte, E.J. Calvo, W. Schuhmann, Precursor sites for localised corrosion on lacquered tinplates visualised by means of alternating current scanning electrochemical microscopy, Electrochim. Acta 48 (2003) 1115-1121.

33. K. Eckhard, H. Shin, B. Mizaikoff, W. Schuhmann, C. Kranz, Constant-distance mode AC-SECM for the visualisation of corrosion pits, Electrochem. Commun. 9 (2007) 1793-1797.

34. K. Eckhard, T. Erichsen, M. Stratmann, W. Schuhmann, Frequency-dependent alternating-current scanning electrochemical microscopy (4D AC-SECM) for local visualisation of corrosion sites, Chem. Eur. J. 14 (2008) 3968-3976.

35. D. Ruhlig, W. Schuhmann, Spatial imaging of $\mathrm{Cu}^{2+}$-ion release by combining alternating current and underpotential stripping mode scanning electrochemical microscopy, Electroanalysis 19 (2007) 191-199.

36. D. Ruhlig, H. Gugel, A. Schulte, W. Theisen, W. Schuhmann, Visualization of local electrochemical activity and local nickel ion release on laser-welded NiTi/steel joints using combined alternating current mode and stripping mode SECM, Analyst 133 (2008) 1700-1706.

37. J.J. Santana, M. Pähler, W. Schuhmann, R.M. Souto, Direct evidence of early blister formation in polymer-coated metals from exposure to chloride-containing 
electrolytes by alternating-current scanning electrochemical microscopy (4D ACSECM), Electrochem. Commun., submitted, ref.

38. M.M. Antonijević, S.M. Milić, M.B. Petrović, Films formed on copper surface in chloride media in the presence of azoles, Corros. Sci. 51 (2009) 1228-1237.

39. S.L. Cohen, V.A. Brusic, F.B. Kaufman, G.S. Frankel, S. Motakef, B. Rush, X-ray photoelectron spectroscopy and ellipsometry studies of the electrochemically controlled adsorption of benzotriazole on copper surfaces, J. Vac. Sci. Technol. A 8 (1990) 2417-2424.

40. D.-Q. Zhang, L.-X. Gao, G.-D. Zhou, Inhibition of copper corrosion in aerated hydrochloric acid solution by heterocyclic compounds containing a mercapto group, Corros. Sci. 46 (2004) 3031-3040.

41. C. Monticelli, G. Brunoro, A. Frignani, Inhibitive action of some Schiff bases and amines towards the corrosion of copper in an aqueous alcoholic medium, Surf. Coat. Technol. 27 (1986) 175-186.

42. R.M. Souto, V. Fox, M.M. Laz, M. Pérez, S. González, Some experiments regarding the corrosion inhibition of copper by benzotriazole and potassium ethyl xanthate, J. Electroanal. Chem. 411 (1996) 161-165.

43. M. Scendo, Potassium ethyl xanthat as corrosion inhibitor of copper in chloride solutions, Corros. Sci. 47 (2005) 1738-1749.

44. R.M. Souto, M.M. Laz, S. González, X-Ray photoelectron spectroscopy and electrochemical studies on the interaction of potassium ethyl xanthate with metallic copper, J. Phys. Chem. B 101 (1997) 508-511.

45. B. Ballesteros Katemann, A. Schulte, E.J. Calvo, M. Koudelka-Hep, W. Schuhmann, Localised electrochemical impedance spectroscopy with high lateral resolution by means of alternating current scanning electrochemical microscopy, Electrochem. Commun. 4 (2002) 134-138.

46. A. Schulte, S. Belger, W. Schuhmann, Imaging localised corrosion of NiTi shape memory alloys by means of alternating current scanning electrochemical microscopy (AC-SECM), J. Mater. Sci. Eng. A. 378 (2004) 523-526.

47. C. Kranz, M. Ludwig, H.E. Gaub, W. Schuhmann, Lateral deposition of polypyrrole lines by means of the scanning electrochemical microscope, Adv. Mater. 7 (1995) 38-40. 
48. A.J. Bard, F.-R.F. Fan, M. Mirkin, Scanning electrochemical microscopy. In: “Electroanalytical Chemistry”, Vol. 18 (A.J. Bard, Ed.). Marcel Dekker, New York, 1994, p. 243.

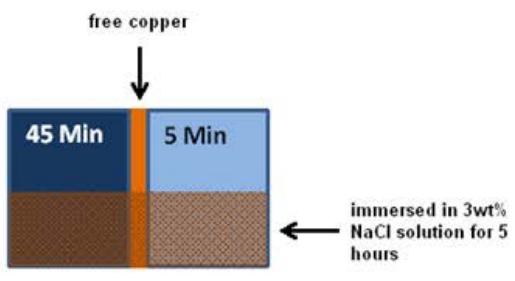

(A)

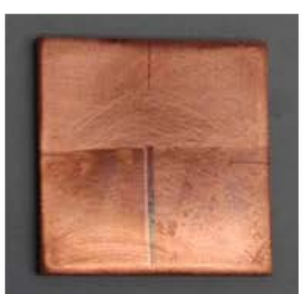

(B)

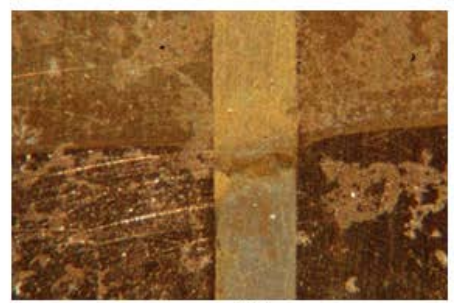

(C)

Figure 1: (A) Sketch depicting sample preparation. The sample is pre-treated in the corresponding inhibitor-containing solution for either 5 or $45 \mathrm{~min}$, leaving at the centre a narrow strip of untreated copper. The persistence of the formed inhibitor films is tested through the immersion of half of the sample in $3 \mathrm{wt} \% \mathrm{NaCl}$ solution for 5 hours. Every sample presents 6 regions of different electrochemical activity. (B) Photograph of a typical sample used in this work. The pre-treatment of the freshly polished copper sample was performed in $1 \mathrm{mM}$ BTAH. (C) Microphotograph taken at the centre of photograph given in (B), which is the area scanned for the obtention of $3 \mathrm{D}$ images using AC-SECM.

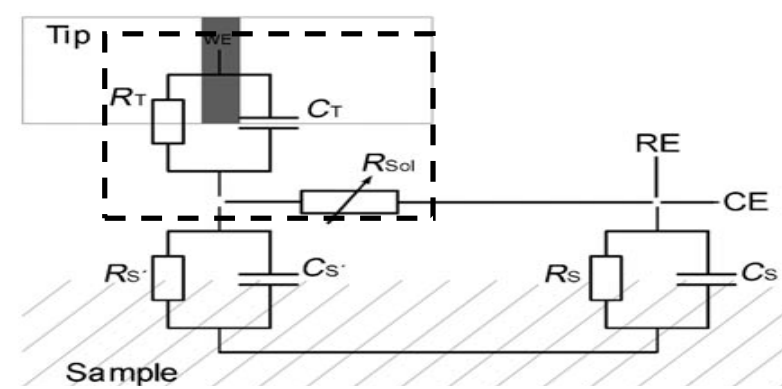

Figure 2: Simplified equivalent circuit representing the impedance behaviour of a large conductive sample in contact with an electrolyte [47]. $R_{T}$ and $C_{T}$ are resistance and capacitance of the tip, respectively; $R_{s o l}$ is the solution resistance; $R_{S}$ and $C_{S}$ are the resistance and capacitance of the sample, respectively; $R_{S}$, and $C_{S}$, are the local resistance and capacitance of the sample at the specific area covered by the AC-SECM tip. 

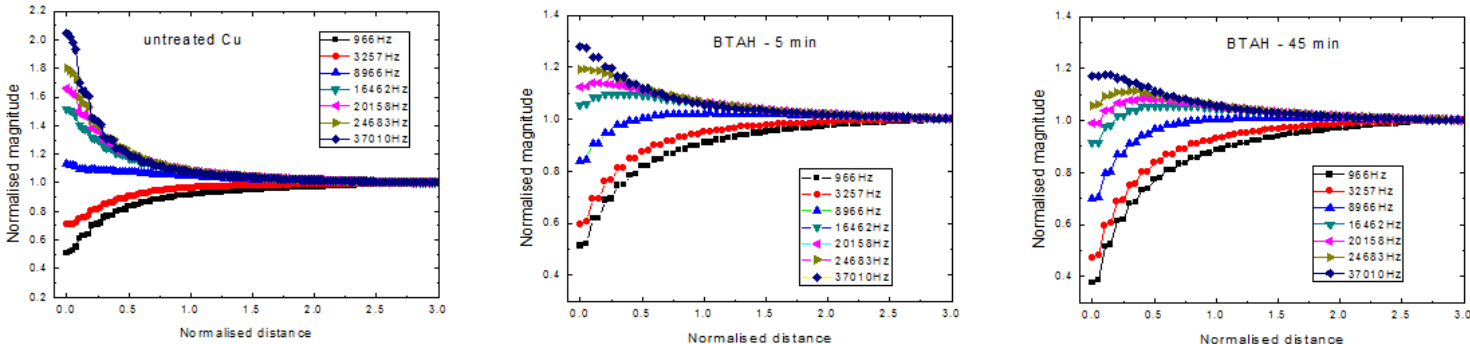

Figure 3: Normalized approach curves towards a Cu/BTAH (45 min pre-treatment) untreated $\mathrm{Cu}-\mathrm{Cu} / \mathrm{BTAH}$ (5 min pre-treatment) system immersed in $1 \mathrm{mM} \mathrm{Na} 2 \mathrm{SO}_{4}$ solution with a $25 \mu \mathrm{m}$ Pt electrode. The sample was left at its spontaneous open-circuit potential in the electrolyte. The excitation signal amplitude was $100 \mathrm{mV}_{\mathrm{pp}}$ and frequencies are indicated in the figure.
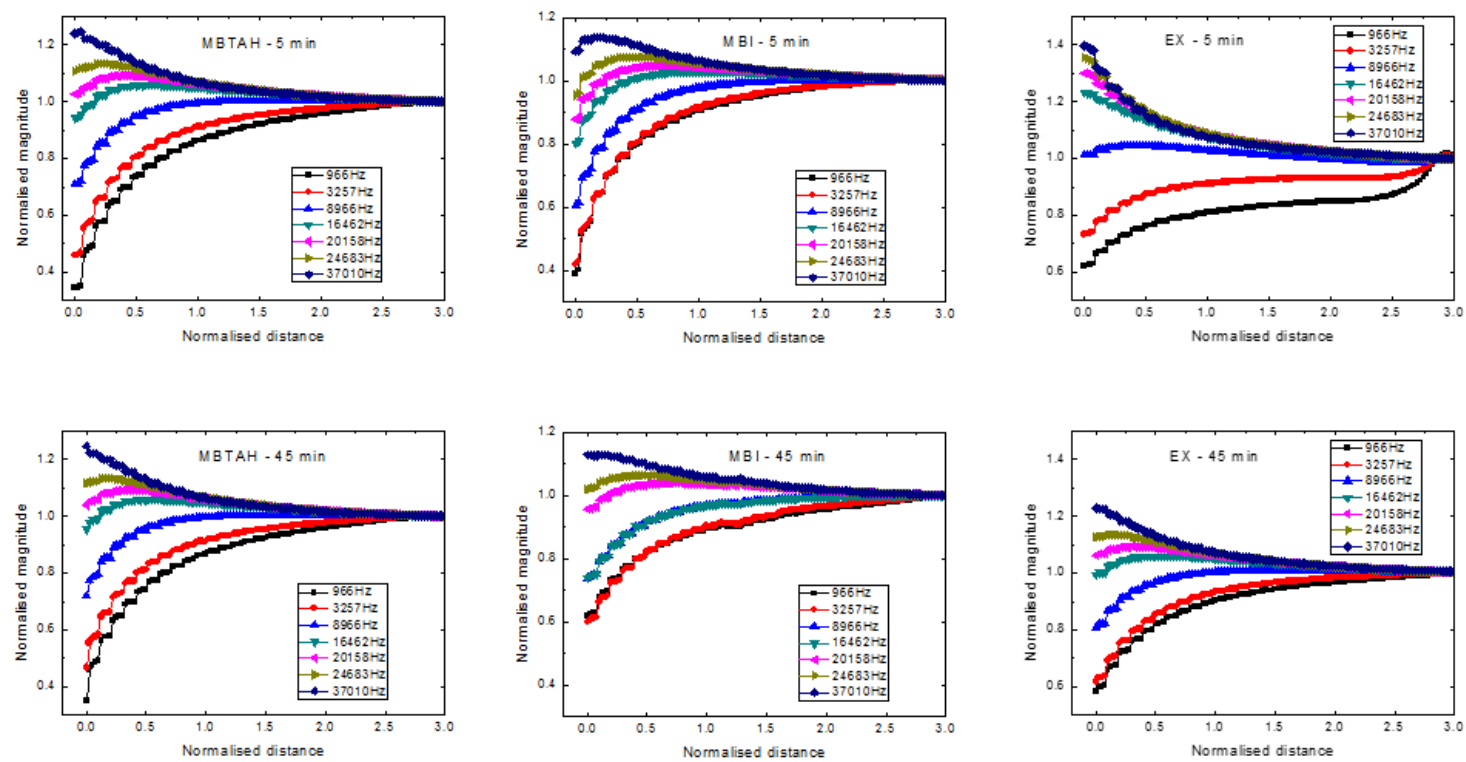

Figure 4: Normalized approach curves towards Cu/MBTAH (5 and 45 min pretreatments), $\mathrm{Cu} / \mathrm{MBI}$ (5 and 45 min pre-treatments), and $\mathrm{Cu} / \mathrm{EX}$ (5 and 45 min pretreatments) systems immersed in $1 \mathrm{mM} \mathrm{Na} \mathrm{SO}_{4}$ solution with a $25 \mu \mathrm{m}$ Pt electrode. The samples were left at their spontaneous open-circuit potential in the electrolyte. The excitation signal amplitude was $100 \mathrm{mV}_{\mathrm{pp}}$ and frequencies are indicated in the figure.

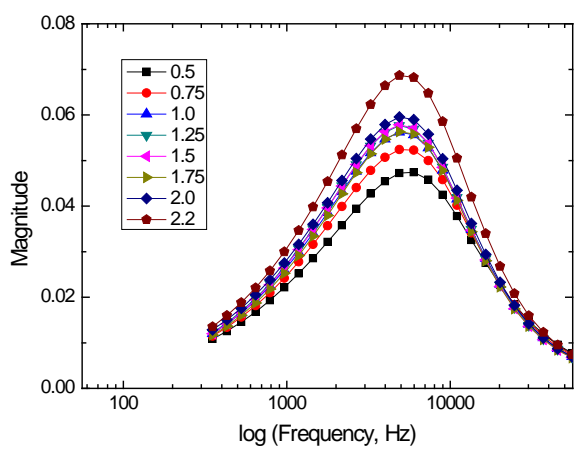

Figure 5: Variation of the current magnitude with the frequency of the AC excitation signal taken from the approach curves for various $z / r$ ratios as indicated in the figure. The substrate was copper pre-treated with BTAH for $30 \mathrm{~min}$. Test electrolyte: $1 \mathrm{mM}$ $\mathrm{Na}_{2} \mathrm{SO}_{4}$. 


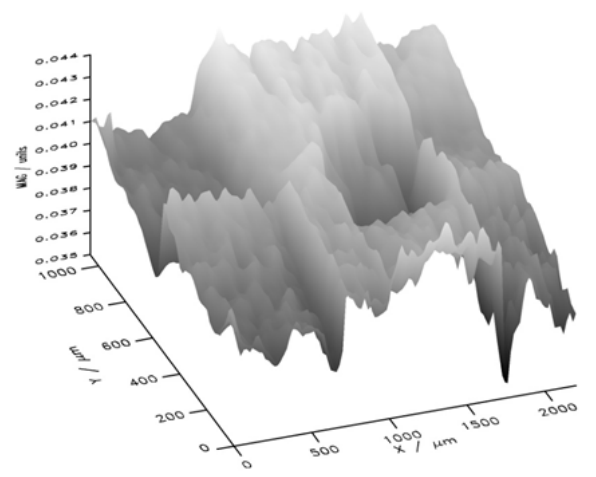

(A)

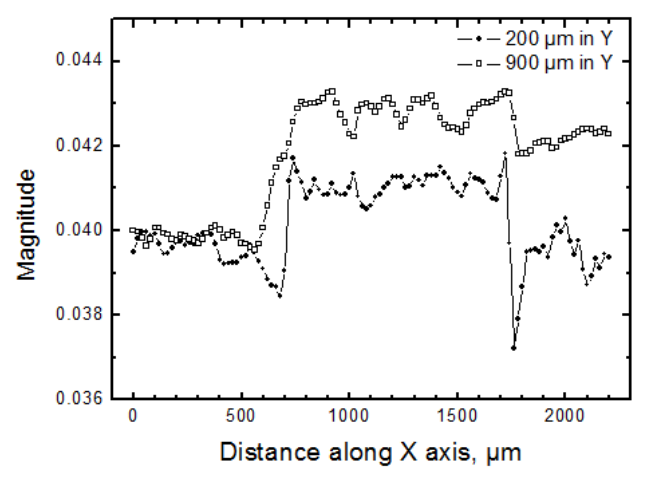

(B)

Figure 6:

AC-SECM measurement on a Cu/BTAH (45 min pre-treatment) - untreated $\mathrm{Cu}$ $\mathrm{Cu} / \mathrm{BTAH}$ (5 min pre-treatment) system. The different regions on the sample may be found as we move from left to right in the maps. The bottom half of the image corresponds to the same regions after exposure to $3 \mathrm{wt} \% \mathrm{NaCl}$ solution for 5 hours. (A) AC-SECM global map depicting the six regions present in the sample; and (B) line scans extracted from the array scan in (A) for the $Y$ coordinates given in the figure. Tipsubstrate distance $=12 \mu \mathrm{m}, f=15650 \mathrm{~Hz}, c=1 \mathrm{mM} \mathrm{Na}_{2} \mathrm{SO}_{4}$.

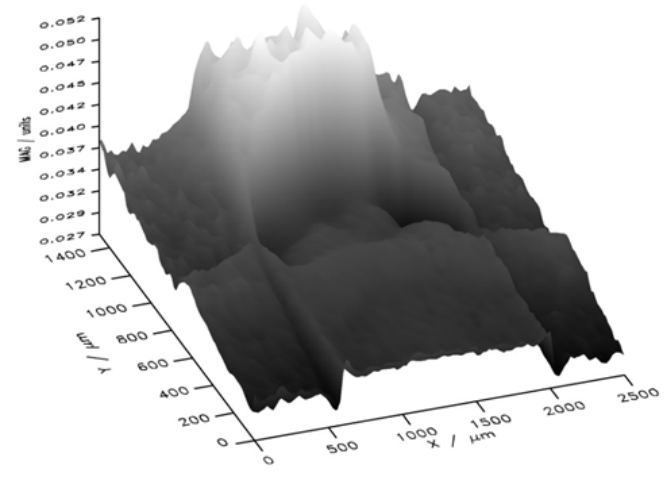

(A)

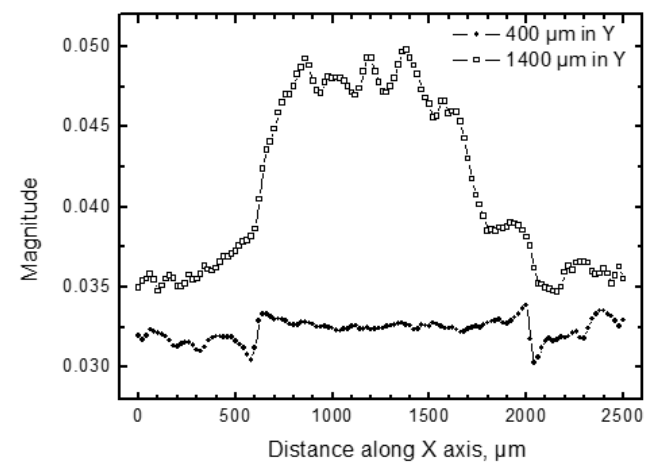

(B)

Figure 7:

AC-SECM measurement on a Cu/MBTAH (45 min pre-treatment) - untreated Cu $\mathrm{Cu} / \mathrm{MBTAH}$ (5 min pre-treatment) system. The different regions on the sample may be found as we move from left to right in the maps. The bottom half of the image corresponds to the same regions after exposure to $3 \mathrm{wt} \% \mathrm{NaCl}$ solution for 5 hours. (A) AC-SECM global map depicting the six regions present in the sample; and (B) line scans extracted from the array scan in (A) for the $Y$ coordinates given in the figure. Tipsubstrate distance $=12 \mu \mathrm{m}, f=15650 \mathrm{~Hz}, c=1 \mathrm{mM} \mathrm{Na}_{2} \mathrm{SO}_{4}$. 


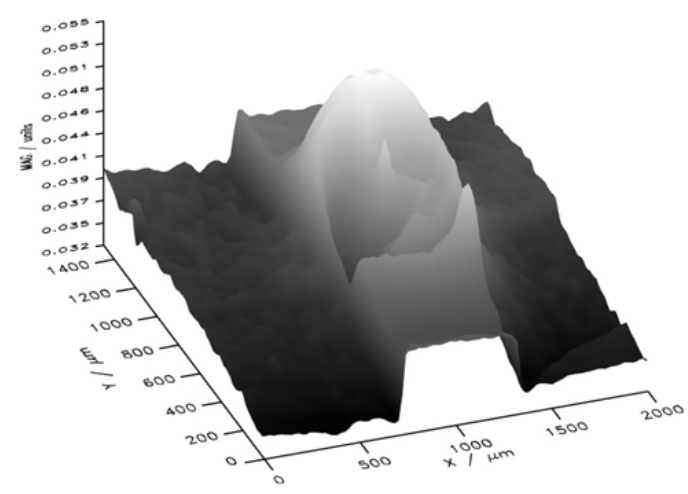

(A)

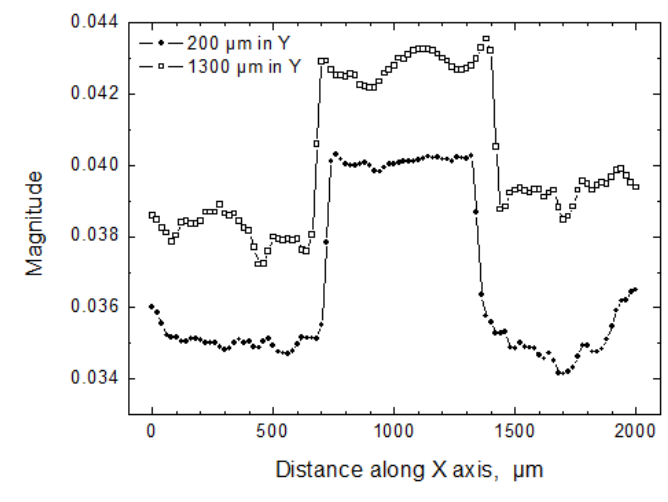

(B)

Figure 8:

AC-SECM measurement on a Cu/MBI (5 min pre-treatment) - untreated $\mathrm{Cu}-\mathrm{Cu} / \mathrm{MBI}$ (45 min pre-treatment) system. The different regions on the sample may be found as we move from left to right in the maps. The upper half of the image corresponds to the same regions after exposure to $3 \mathrm{wt} \% \mathrm{NaCl}$ solution for 5 hours. (A) AC-SECM global map depicting the six regions present in the sample; and (B) line scans extracted from the array scan in (A) for the $Y$ coordinates given in the figure. Tip-substrate distance $=$ $12 \mu \mathrm{m}, f=15650 \mathrm{~Hz}, c=1 \mathrm{mM} \mathrm{Na}_{2} \mathrm{SO}_{4}$.

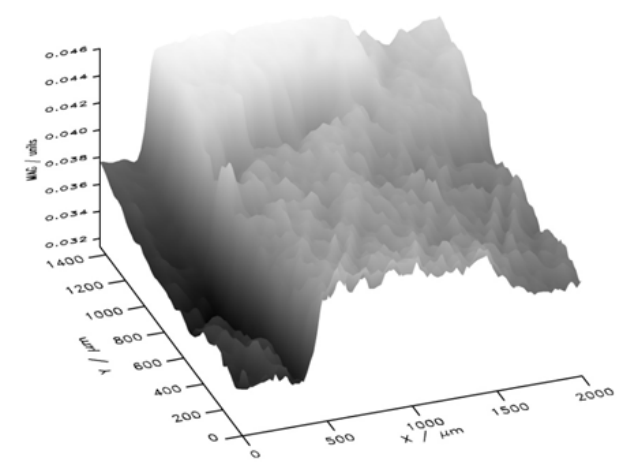

(A)

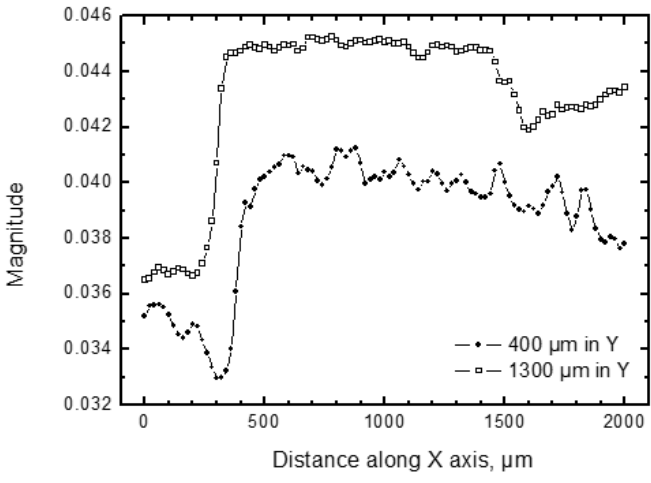

(B)

Figure 9:

AC-SECM measurement on a Cu/EX (45 min pre-treatment) - untreated $\mathrm{Cu}$ - Cu/EX (5 min pre-treatment) system. The different regions on the sample may be found as we move from left to right in the maps. The bottom half of the image corresponds to the same regions after exposure to $3 \mathrm{wt} \% \mathrm{NaCl}$ solution for 5 hours. (A) AC-SECM global map depicting the six regions present in the sample; and (B) line scans extracted from the array scan in (A) for the $Y$ coordinates given in the figure. Tip-substrate distance $=$ $12 \mu \mathrm{m}, f=15650 \mathrm{~Hz}, c=1 \mathrm{mM} \mathrm{Na}_{2} \mathrm{SO}_{4}$. 